\title{
Design of a Power Management System using Nano-power Boost Charger for a Quadcopter- based Air Quality Monitoring Device
}

\author{
John Joel F. Martinez ${ }^{1}$, Shearyl U. Arenas ${ }^{1}$ and Angelo John H. Francisco ${ }^{1}$ \\ College of Engineering and Architecture -Electronics Engineering, Technological Institute of the Philippines, \\ Quezon City, Philippines ${ }^{1}$
}

\begin{abstract}
In this paper, a design of a power management system for a quadcopter-based air quality monitoring device was proposed. The design aims to extend the operating time of the battery. It employs a nanopower boost charger as a means of efficiently harvesting the ambient solar energy. The design also utilize maximum power point tracking (MPPT) algorithm to maximize the extracted power. Boost converter for single cell batteries was used to translate voltage output into the required voltages for multiple loads.
\end{abstract}

Keywords: air quality; wireless sensor networks; quadcopter; solar; power management; energy harvesting.

\section{INTRODUCTION}

The availability of low powered, miniature components like processors, radios and sensors paved the way for an increased interest for wireless sensor networks. The idea of internet of things (IoT) was born in parallel with the said interest [1]. Wireless sensor networks are described as a network of nodes that senses a certain environment.

A network of these sensors provides a stream of data regarding certain parameters needed to be measured. An example of this is the measurement of air quality, and how data gathering affects projects that promote better air quality. Gathering air quality data through IoT can promote social and environmental changes if they are accumulated properly and analyzed correctly [2]. Information from air quality monitoring can be used when checking the air quality of a certain area, or improving people's well-being. The network of sensor helps to the cause of such data-gathering methods.

In these kinds of low-powered sensing devices, a battery pack and limited-capacity alternative energy source are used, but there are other requirements to be considered. Aside from the need to convert the voltage level to a precise DC value, and energy storage for the rail, there are also important matters to consider like energy saving, quality of output with respect to fast load transients, electromagnetic compatibility, and protection aspects. These comprise the power management aspect of a system's power rail [3].

These devices will require power management that will ensure continuous operations, given that AC-powered or battery replacement is not a feasible option [4]. Harvesting energy from ambient sources, like solar energy, thermoelectric energy, mechanical energy, and bioelectric energy, have been targets for powering, or recharging such devices for its continuous, perpetual operation.

Power management techniques are now being researched and developed as such circuits are important to be rightfully integrated in the implementation of a sensor node. Bridging the gap, from the development of these techniques up to its implementation to a sensor, can now become a focus for those interested in its application. Energy-harvesting devices would not behave as a plug-in module for a system; as such converters need better matching with the processors or communications module.

\section{DESIGN CONSIDERATION}

\section{A. System Block Diagram}

The design of the power management system is an application specific, intended as a subsystem for an air quality measuring device. The entirety of the system, presented below, aims to take ambient air, and provide a numerical value on the level of pollution within that air. 


\section{IJIREEICE \\ International Journal of Innovative Research in Electrical, Electronics, Instrumentation and Control Engineering}

ISO 3297:2007 Certified

Vol. 5, Issue 4, April 2017

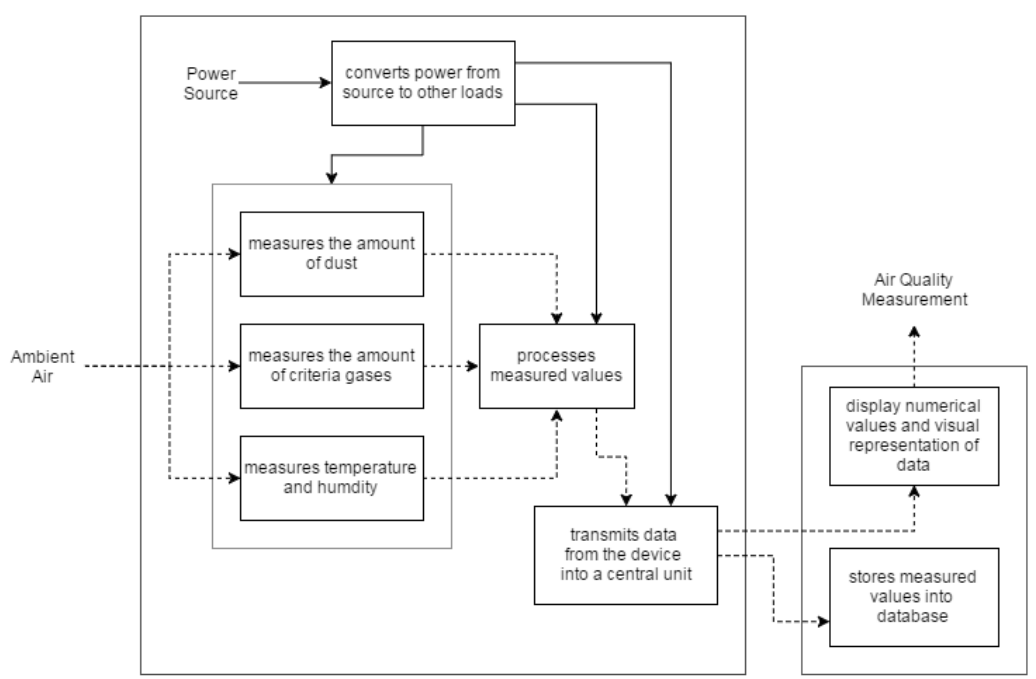

Fig. 1. Air Quality Monitorind Device block diagram

B. Estabilishing Functions

The system is envisioned to be a mobile device, therefore requiring a battery, which supplies energy to the system. It utilizes another subsystem that converts the voltage level from the power source, into a level more suitable to the requirements of the multiple loads. The conversion should be efficient to avoid power losses along the subsystem levels. With an aim at prolonging battery life, which is found to be needed on such measuring devices, another subsystem is added. The battery-prolonging circuit is an energy harvester, which uses ambient energy and converts it to electrical energy to be used by the device. The figure below shows the input-output relationship of the whole system.

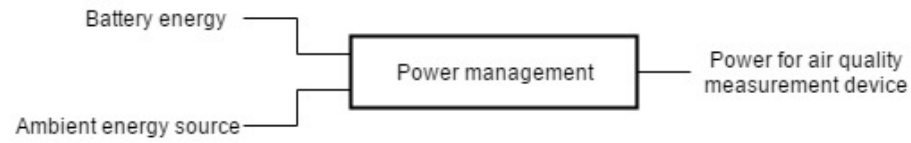

Fig. 2. Block Box model for Power Management System

We break the intended power management circuit to subsystems that are based on the design considerations for energy harvester circuits, in addition to design considerations for power converter design.

First is the DC-DC input stage converter that steps-up the harvested energy. Maximum power point tracking is a feature added for the circuit to optimize the harvesting of the energy. The harvested energy is then going to be used in recharging the battery, which is the main power source, for the continuous usage of the sensing device. A battery charger with protection, which bridges the harvested energy and charges the battery, is needed. A power converter, meanwhile, which converts the voltage level from the battery pack, is at the output stage of the power management circuit. The block diagram stating the intended function is drawn below:

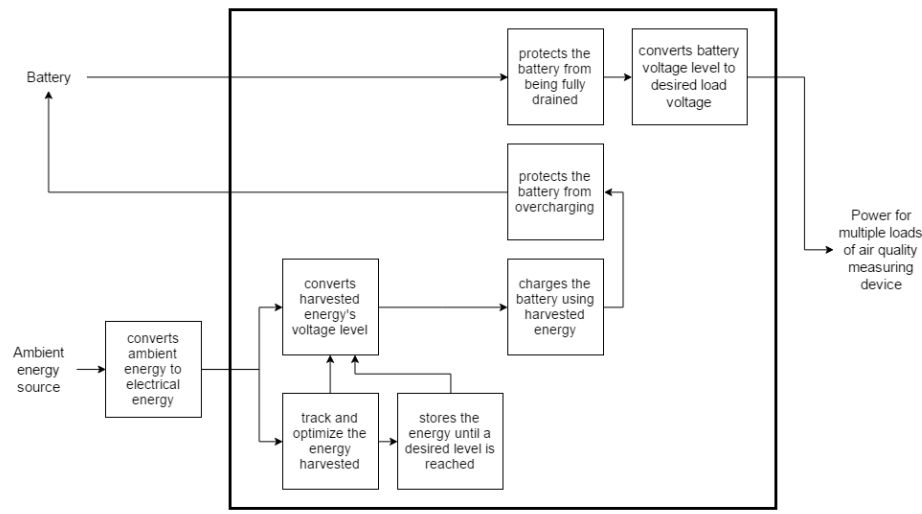

Fig. 3. Functional Block Diagram for Power Management System 
C. Switching Converters for Low-Power Applications

In today's time, power management units for portable products require high power conversion efficiency, fast line/load transient response, and small power module volume [10]. With a variation in voltage levels among different submodules of such portable products, there are different designs of power converters that have been developed over the years.

For power electronic systems, having high efficiency coupled with high power density is a way to reduce the size and weight of the system, and is used to lower the operating cost of systems by avoiding loss [5]. Power electronics provides efficient transfer of power in DC-DC systems. The controller allows management of the power conversion to be highly efficient with high power density.

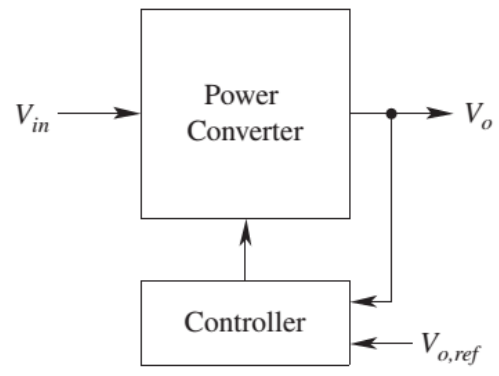

Fig. 4. Block diagram for a basic Regulated Low-Voltage DC Power supply

\section{Energy Harvesting}

The four main ambient energy sources available are: mechanical, thermal, radiant and biochemical sources. Under mechanical, energy can be scavenged from stresses-strains, or from vibrations; from thermal sources, are thermal gradient or variations; while from radiant sources, is the solar power, infrared or RF sources [4]. The electricity to be generated from the harvesting unit will power the systems and charge the energy storage. The power management unit which includes an energy harvesting circuit then performs tasks such as: impedance matching for maximum energy harvesting, charging of energy storage, routing power, converting the correct voltage for the system, and monitoring current flows and voltage generation [6]. A proper integration of these functions can help increase efficiency in performing the said tasks. Power management also allows putting the system in a low-power state while the battery is recharging. Additional feature includes maximum power point tracking, which ensures that the most energy is harvested from an alternate source. An energy harvesting using small photovoltaic cells has been the proposed use for perpetually operating wireless sensor nodes. This is because of its established technology and rapid development over the years [7], making it a reasonable technical solution.

There are three building blocks of the circuit architecture of a solar harvester circuit [8].

- DC-DC Input stage - Most of the MPPT systems employ switch-mode power converter topologies, which are driven by pulse-width modulated signal generated in control.

- MPP Tracker - It estimates the Maximum Power Point using open-loop configuration without interference from photovoltaic module. The implementation used is a single low-power comparator that compares the current PV module voltage to the estimated MPP generated using the PWM signal.

- Output DC-DC Converter - The output converter that will deliver power to the load needs to match the input voltage of the load. This allows improving the efficiency.

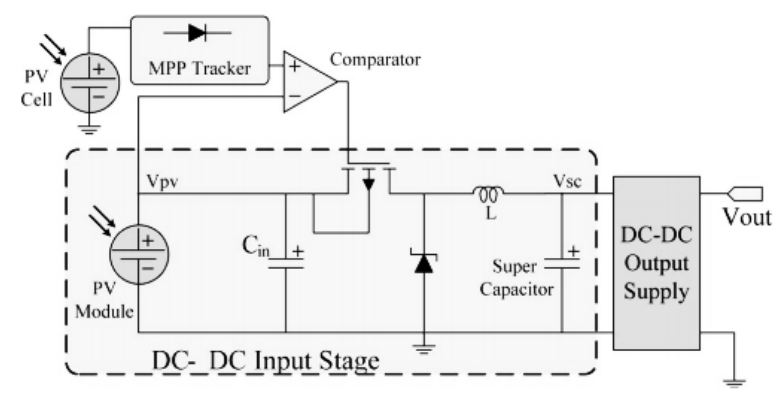

Fig. 5. Schematic Diagram of Solar Harvester [8] 


\section{ISO 3297:2007 Certified}

Vol. 5, Issue 4, April 2017

A recent control algorithm, namely maximum power point tracking, is developed to maximize the advantage of the available solar energy. Because of this, the direction to utilize solar energy in order to maximize the efficient use of energy harvesting is conducted. The operation of MPPT is to adjust photovoltaic interfaces so the operating characteristics of the load and the photovoltaic array match at maximum power point [9].

\section{DESIGN APPROACH}

The bq25570 integrated circuit is used in efficiently harvesting power generated from high impedance sources like photovoltaic source. The IC is well suited for low-power applications designed to efficiently harvest energy from ambient sources. It features management technique to protect the battery from overcharging, or from being depleted beyond the safe limits by system load, and maximum power point tracking, which maximizes the power extracted from the energy source. In this Nanopower boost charger circuit, the maximum power point is at $70 \%-80 \%$ of the opencircuit voltage of the solar panel [9].

LT1308 is a boost converter for single cell batteries that can provide power even at as low as $1 \mathrm{~V}$. It can provide $5 \mathrm{~V}$ from a single cell lithium ion battery. It operates at $600 \mathrm{kHz}$ switching frequency which could make components smaller. It also provides fast response to line and load regulation.

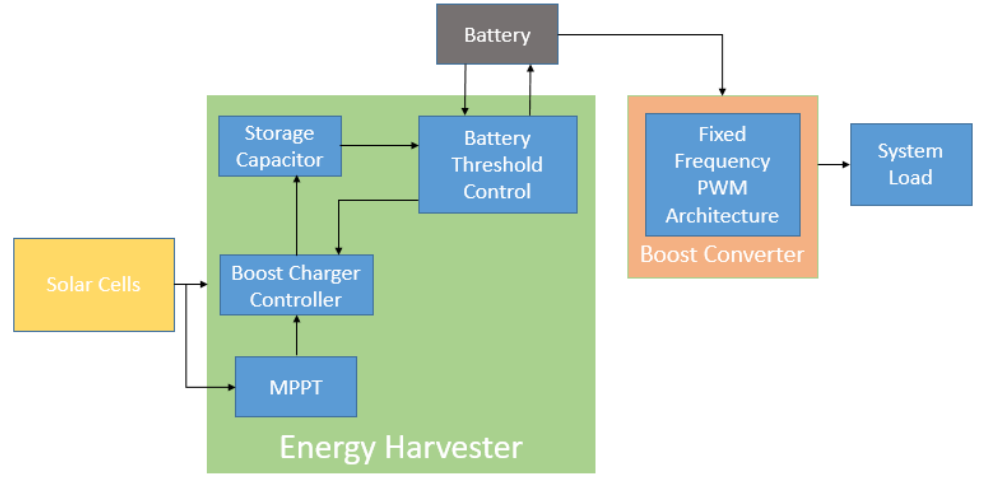

Fig. 6. Power Management Functional Block Diagram

The circuit uses a voltage divider network in programming voltage levels for different operations. To prevent rechargeable batteries from being deeply discharged and damaged, and to prevent completely depleting charge from a capacitive storage element, the boost charger has an internally set under voltage (VBAT_UV) threshold plus an internal hysteresis voltage (VBAT_UV_HYST). The VBAT_UV threshold voltage when the battery voltage is decreasing is internally set to $1.95 \mathrm{~V}$ (typical). To prevent rechargeable batteries from being exposed to excessive charging voltages and to prevent over charging a capacitive storage element, the over-voltage (VBAT_OV) threshold level must be set using external resistors. This is also the voltage value to which the charger will regulate the VSTOR/VBAT pin when the input has sufficient power. The charger allows the user to set a programmable voltage independent of the overvoltage and under voltage settings to indicate whether the VSTOR voltage (and therefore the VBAT voltage when the PFET between the two pins is turned on) is at an acceptable level. When the battery voltage is decreasing the threshold is set by

$$
\frac{\mathrm{R}_{\mathrm{OK} 2}}{\mathrm{R}_{\mathrm{OK} 1}}=\frac{\mathrm{VBA}_{\mathrm{OK}_{\mathrm{PROG}}}}{\mathrm{V}_{\mathrm{BIAS}}}-1
$$

To set the proper output regulation voltage and input voltage power good comparator, the external resistors must be carefully selected.

The OUT regulation voltage is then given by:

$$
\frac{\mathrm{R}_{\text {OUT } 2}}{\mathrm{R}_{\text {OUT } 1}}=\frac{\mathrm{V}_{\text {OUT }}}{V_{\text {BIAS }}}-1
$$

All capacitors to be used need to be low leakage. For the capacitance in VIN_DC, the energy from the harvester input is initially stored in the capacitor. The value for this capacitance should be scaled depending on the capacitance of the energy source, but a minimum value of $4.7 \mu \mathrm{F}$ is recommended. The output capacitor depends on transient response behavior, and when the value is lower, the larger the ripple becomes. The recommendation for the capacitance is at 22 $\mu \mathrm{F}$. 


\section{IJIREEICE \\ International Journal of Innovative Research in Electrical, Electronics, Instrumentation and Control Engineering \\ ISO 3297:2007 Certified \\ Vol. 5, Issue 4, April 2017}

If there are large, fast system load transients and/or the storage element has high resistance, then the CSTOR capacitors may momentarily discharge below the VBAT_UV threshold in response to the transient. The CSTOR capacitors may further discharge below the VSTOR_CHGEN threshold and cause the bq25570 to enter Cold Start.

$$
\mathrm{C}_{\mathrm{STOR}}=\frac{\mathrm{I}_{\mathrm{L}} \times \mathrm{t}_{1}}{\mathrm{~V}_{\mathrm{hi}}-\mathrm{V}_{\mathrm{lo}}}
$$

An inductor for the boost charger must have a peak current capability of $>300 \mathrm{~mA}$ to maintain high efficiency. Its internal circuitry is designed with a nominal inductance of $22 \mu \mathrm{H} \pm 20 \%$. For the buck converter, the inductor must have a peak current capability of $200 \mathrm{~mA}$, as its internal circuitry is designed to control switching behavior with a nominal frequency of $10 \mu \mathrm{H} \pm 20 \%$

$$
L=\frac{V_{\text {in }} \times\left(V_{\text {out }}-V_{\text {in }}\right)}{\Delta i_{\mathrm{L}} \times f_{s} \times V_{\text {ouT }}}
$$

The critical components for the single cell boost converter are shown next. The computations are based from the datasheet of the LT1308 boost converter specified by the manufacturer.

For the boost converter, the inductor must have a peak current capability of $200 \mathrm{~mA}$, as its internal circuitry is designed to control switching behavior with a nominal frequency of $10 \mu \mathrm{H} \pm 20 \%$. The suggested choice based on the datasheet for the LT1308B has a range of $2 \mu \mathrm{H}$ to $20 \mu \mathrm{H}$.

$$
\mathrm{L}=\frac{\mathrm{V}_{\text {in }} \times\left(\mathrm{V}_{\text {out }}-\mathrm{V}_{\text {in }}\right)}{\Delta \mathrm{i}_{\mathrm{L}} \times \mathrm{f}_{\mathrm{S}} \times \mathrm{V}_{\text {OUT }}}
$$

The inductor fits the value within the range given from the IC's datasheet. With frequency set at $13 \mathrm{kHz}$, the formula used for the LC combination is:

$$
f=\frac{1}{2 \pi \sqrt{L C}}
$$

The RC compensation sets the frequency limit allowed in the IC, which acts as a low-pass filter and shuts high frequency noise down. The compensation frequency is set at $10.3 \mathrm{kHz}$. Computing for the RC combination,

$$
\mathrm{f}=\frac{1}{2 \pi \mathrm{RC}}
$$

The shunt input capacitor affects the stability of the output voltage regulation to maintain it below the compensation frequency early computed. Mostly used for noise suppression, it has typical value of $0.1 \mu \mathrm{F}$.

To set the output voltage, the circuit uses voltage divider feedback which has an internally set reference bias voltage of $1.21 \mathrm{~V}$. Solving for appropriate values of voltage divider ratio to produce the expected output using equation (2), we get

$$
\frac{\mathrm{R}_{\text {OUT } 2}}{R_{\text {OUT } 1}}=\frac{V_{\text {OUT }}}{1.21}-1
$$

\section{EXPERIMENTAL RESULTS}

The simulation shows the response of the circuit showing the input power given by the source voltage (red) and the input current (cyan) drawn from the battery while it is simultaneously charged by the solar cells and discharged to the load, and the output power represented by the output voltage (green) which satisfies the requirements of the load circuit and the output current (blue).

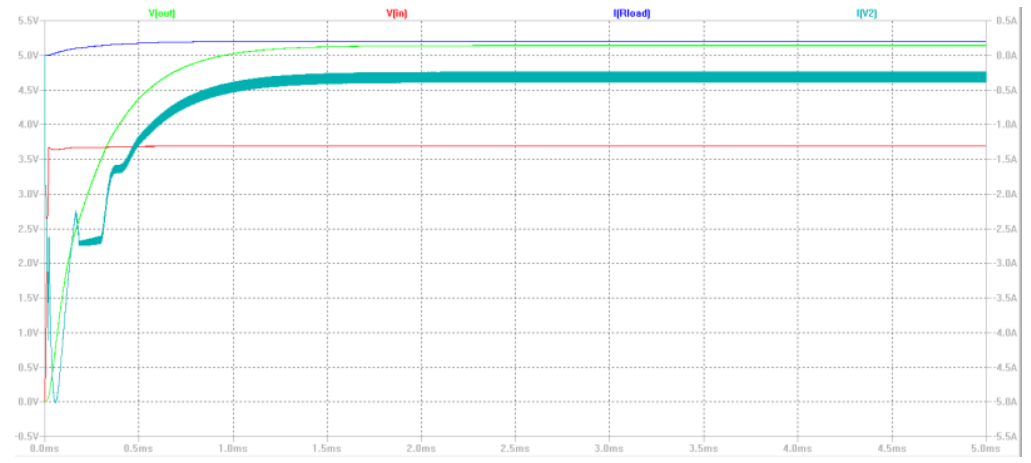

Fig. 7. Simulation result using LTSPICE

All the parameters achieve a stable condition at $1 \mathrm{~ms}$ which suggests that constant supply is given to the load at the nominal battery voltage $(3.7 \mathrm{~V})$ which is the average lithium battery state and the load circuit is drawing constant current at a maximum of $200 \mathrm{~mA}$. 
Vol. 5, Issue 4, April 2017

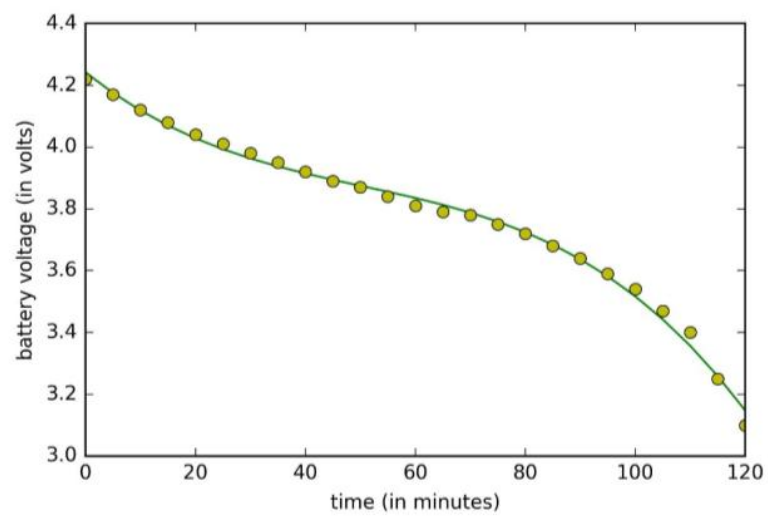

Fig. 8. Battery Life Evaluation without Nanopower Boost Charger

Figure 7 shows the battery reaching the minimum threshold capacity of $3.3 \mathrm{~V}$ at almost 2 hours which is where the battery is at low battery condition thus, the design can only last until 115 minutes when no energy harvesting scheme is employed without the Nanopower boost charger circuit.

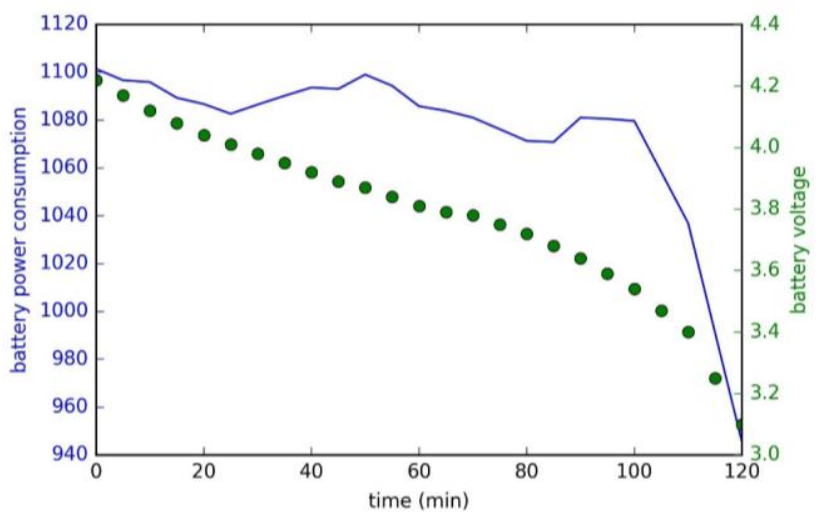

Fig. 9. Plot of Battery Power Consumption and Batter Voltage

The plots above shows how much power is being consumed from the battery per unit time as it goes from full level condition to low battery charge condition. It shows maximum values at nominal range and increases again when the battery is low voltage which increased the discharge rate that drained the energy of the battery faster at low voltage conditions. Thus, the power consumption of the battery increases when the battery voltage is nearly approaching low which is the reason why the battery drains faster during this condition. To evaluate the effectiveness of the energy harvesting design, the nanopower boost charger design with the solar panel is attached to the battery and the boost converter. The parameters we are looking into for the testing are the light intensity using a lux meter, the voltage at the maximum power point tracked by the design reflected in the terminals of the solar panel, the charging current produced by the solar panel and the battery voltage which is monitored while the system load is connected.

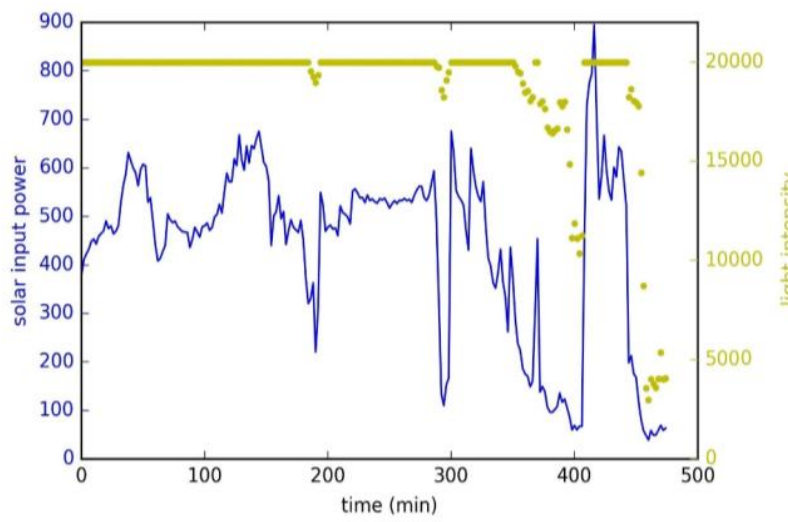

Fig. 10. Plot of light instensity and solar input power with respect to time 
The result shows that the battery life utilizing the Nanopower boost charger design was able to extend the battery life until 8 hours and 20 minutes under varying light conditions displayed above with the solar power input being a factor in achieving this objective and thus, the power management design was able to be used in achieving the specific objective of extending the battery life of the system. This is comparatively longer than the system without the Nanopower boost charger design draining the battery after 1 hour and 55 minutes.

In an effort to understand the evaluation procedure, a breakdown analysis of the data is presented beginning with analyzing the behavior of the solar input power in relation to the illuminance at the certain period of time. Most of the measured light intensity bounded at 20,000 (equipment limitations of lux meter) signifies full daylight or direct sunlight which has values ranging from 25,000 to 100,000 lux.

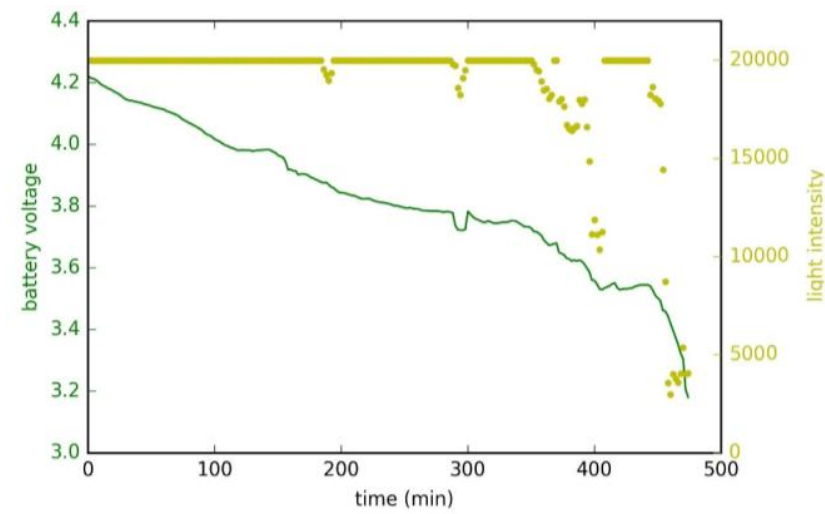

Fig. 11. Plot of battery voltage and light intensity with respect to time

The variations in the light intensity can also be seen in effect to the rate of discharge of the battery causing steeper slopes at times when illumination is low and this can be observed more in the rightmost part of the plot shown below which corresponds to the time approaching sunset where the daylight intensity is getting lower.

The next plot describes how the solar input power aids in extending the battery life showing the flattening of the downward slope as solar input power increases in level at some intervals along the testing data.

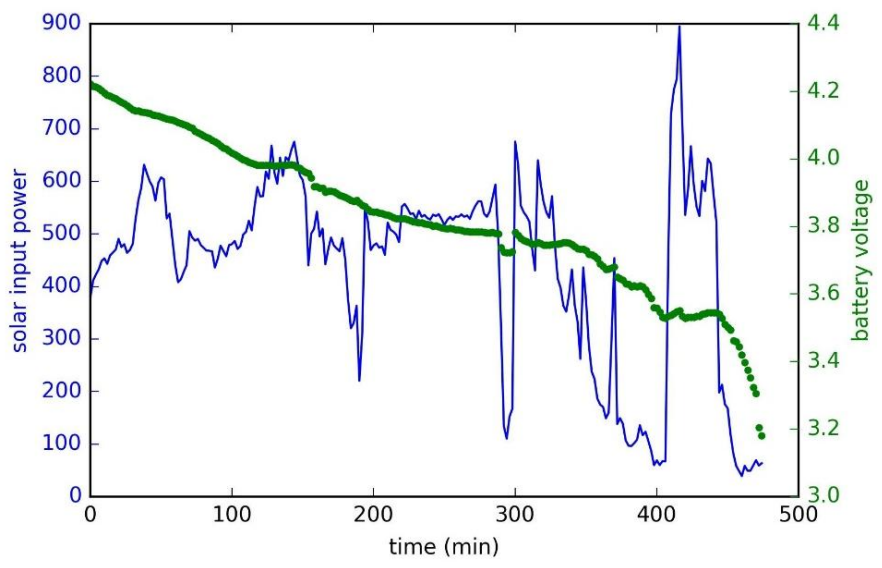

Fig. 12. Plot of battery voltage and solar input power with respect to time

The figure below shows the comparison plot between the battery life with and without the power management design utilizing Nanopower boost charger which shows the test plot with energy harvesting scheme employing the power management design to last longer therefore, the Nanopower boost charger was able to be used in attaining the objective of the design to extend the battery life of the system.

Line regulation is determined by how much the output voltage will vary when the input voltage is set to low line (3.3V) which is the typical under-voltage of a lithium battery according to ANSI C18.3M standards and when it is set to high line $(4.2 \mathrm{~V})$, a fully charged battery voltage. The required values for determining line regulation are the nominal load condition output voltage at low line and high line. The percent line regulation is found to be $2.22 \%$ 


\section{International Journal of Innovative Research in} Electrical, Electronics, Instrumentation and Control Engineering

ISO 3297:2007 Certified

Vol. 5, Issue 4, April 2017

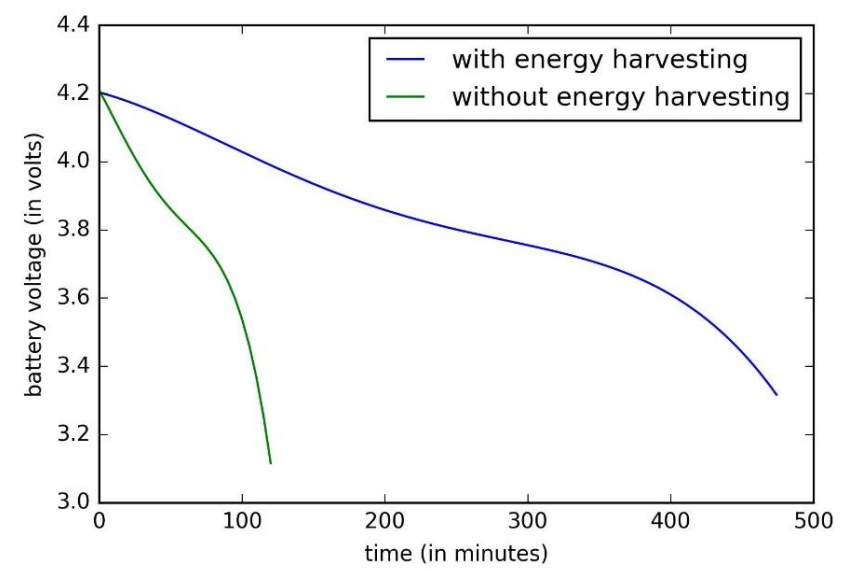

Fig. 13. Plot of battery voltage and solar input power with respect to time

Load regulation tells how much the output voltage change when the load current is varied from the no-load condition to the maximum current rating or usually know as full load condition. The values in evaluating the load regulation are taken at nominal line voltage $(3.7 \mathrm{~V})$ with full load at $200 \mathrm{~mA}$ and no-load at $0 \mathrm{~A}$. The percent load regulation is found to be $1.54 \%$. The mean conversion efficiency is found out to be at $84.10984 \%$.

\section{CONCLUSION}

The purpose of this study was to design a power management system for wireless sensor networks. The design focused on power management solution through extending battery life of the system and efficient power conversion from the source to the loads of the system which comprises of an integration of air quality sensors, microcontroller and communications module. The objective of extending the battery life of the system was achieved using the Nanopower boost charger design employing energy harvesting schemes using solar panels with maximum power point tracking algorithm which optimizes harvested energy. The results show that the battery life of the system was extended from about 1 hour and 55 minutes to about 8 hours and 20 minutes under normal daylight condition. A graphical analysis on parameters that affected the design in attaining this objective was also presented such as light intensity and equivalent solar input power. The objective of efficiently converting power from the source to the loads of the system was achieved by first, modelling the design based on target specifications of conversion efficiency, line regulation and load regulation, choosing the components wisely based on the model, simulating the design using available simulation software, trading off between designs, prototyping and evaluation through experimentation. The results showed the target specifications of the project being met. The conversion efficiency achieved a mean value of $84.109 \%$ and can reach as high as $90.55 \%$ at high line condition. The line and load regulation were achieved with line regulation of the design at $2.22 \%$ and the load regulation at $1.54 \%$.

\section{REFERENCES}

[1] IEC. (2014). Internet of Things: Wireless Sensor Networks. Geneva: International Electrotechnical Commission.

[2] M.J. Rowley. Air quality and the Internet Of Things. Retrieved 2017, from Cisco, The Network: https://newsroom.cisco.com/featurecontent?type=webcontent\&articleId=1757249, 2016.

[3] N. Kulartna, DC Power Supplies: Power Management and Surge Protection for Power Electronics Systems. Boca Raton, FL: CRC Press, 2012.

[4] R. Giaffreda, and A. Somov, Powering IoT Devices: Technologies and Opportunities. Retrieved from IEEE Internet of Things: http://iot.ieee.org/newsletter/november-2015/powering-iot-devices-technologies-and-opportunities.html, 2015.

[5] N. Mohan, Power Electronics - A First Course. John Wiley \& Sons, Inc, 2012.

[6] F. Dostal, New Advances in Energy Harvesting Power Conversion. Retrieved from Analog Dialogue: http://www.analog.com/en/analogdialogue/articles/energy-harvesting-power-conversion.html, 2015.

[7] D. Dondi, D. Brunelli, L. Benini, P. Pavan, A. Bertacchini, \& L. Larcher, Photovoltaic Cell Modeling for Solar Energy Powered Sensor Networks. International Workshop on Advances in Sensors and Interface, 2007.

[8] D. Dondi, A. Betacchini, D. Brunelli, L. Larcher \& L. Benini, Modeling and Optimization of a Solar Energy Harvester System for SelfPowered Wireless Sensor Networks. IEEE Transactions onIndustrial Electronics, 2008.

[9] W. Xiao, N. Ozog, \& W. Dunford, Topology Study of Photovoltaic Interface for Maximum Power Point Tracking. IEEE Transactions on Industrial Electronics, 2007.

[10] Texas Instruments, bq25570 Nano Power Boost Charger and Buck Converter for Energy Harvester Powered Applications, 2015.

[11] M. Huang, \& K. Chen. Single Inductor Multiple Output DC-DC Converters with High Light Load Efficiency and Minimized Cross-Regulation for Portable Devices. IEEE Journal of Solid State Circuits, 2009. 